\title{
IMPERFECT MAINTENANCE IN A GENERALIZED COMPETING RISKS FRAMEWORK
}

\author{
LAURENT DOYEN, ${ }^{*}$ Université Pierre Mendès France Grenoble 2 \\ OLIVIER GAUDOIN,** Institut National Polytechnique de Grenoble
}

\begin{abstract}
In this paper we present a general framework for the modelling of the process of corrective and condition-based preventive maintenance actions for complex repairable systems. A new class of models is proposed, the generalized virtual age models. On the one hand, these models generalize Kijima's virtual age models to the case where both preventive and corrective maintenances are present. On the other hand, they generalize the usual competing risks models to imperfect maintenance actions which do not renew the system. A generalized virtual age model is defined by both a sequence of effective ages which characterizes the effects of both types of maintenance according to a classical virtual age model, and a usual competing risks model which characterizes the dependency between the two types of maintenance. Several particular cases of the general model are derived.
\end{abstract}

Keywords: Point process; reliability; imperfect maintenance; competing risks; virtual age

2000 Mathematics Subject Classification: Primary 60K10; 60G55

Secondary 90B25; 62N05

\section{Introduction}

The dependability of complex repairable systems depends strongly on the efficiency of preventive and corrective maintenance actions. Corrective maintenance (CM), also called repair, is carried out after a failure and is intended to put the system into a state in which it can perform its function again. Preventive maintenance (PM) is carried out when the system is operating and is intended to slow down the wear process and reduce the frequency of occurrence of system failures. Planned PM occurs at predetermined times. Condition-based PM occurs at times which are determined according to the results of inspections and degradation or operation controls. In this study, we focus on condition-based PM. Then CM and PM times are both random and the sequence of maintenance times is a random point process.

The basic assumptions on maintenance efficiency are known as minimal repair or 'as bad as old' (ABAO) and perfect repair or 'as good as new' (AGAN). In the ABAO case, each maintenance leaves the system in the state it was in before maintenance. In the AGAN case, each maintenance is perfect and leaves the system as if it were new. Obviously, reality is between these two extreme cases: standard maintenance reduces failure intensity but does not leave the system as good as new. This is known as imperfect maintenance.

Several models incorporating this type of assumption have already been proposed (see, for example, the review [17]). Most of them, known as repair models, consider only the effect of

Received 22 July 2005; revision received 5 May 2006.

* Postal address: Laboratoire LABSAD, Université Pierre Mendès France Grenoble 2, BP 47-38 040 Grenoble Cedex 9 , France. Email address: laurent.doyen@iut2.upmf-grenoble.fr

** Postal address: Laboratoire LMC, Institut National Polytechnique de Grenoble, BP 53-38 041 Grenoble Cedex 9 , France. Email address: olivier.gaudoin@imag.fr 
CM. For example, the Brown-Proschan model [4] assumes that CM is AGAN with probability $p$ and $\mathrm{ABAO}$ with probability $1-p$, and the virtual age models of [12] assume that the system after $\mathrm{CM}$ is 'younger' than it is before failure. Other models consider only the effect of PM. However, the same kinds of assumption are made for CM or PM, so similar models can be considered to study the effect of both kinds of maintenance. When only one type of maintenance is taken into account, these models are completely defined by the intensity of the maintenance process. The ABAO case corresponds to nonhomogeneous Poisson processes and the AGAN case involves renewal processes.

When both kinds of maintenance are considered, it is necessary to add information about the dependence between PM and CM. The usual competing risks framework provides this information, but it assumes that the PM and CM efficiencies are both perfect. A first attempt to overcome this restrictive assumption was made by Langseth and Lindqvist [14], who applied a competing risks model with maintenance efficiency of the Brown-Proschan type.

In this paper, we present a general framework for modelling the PM-CM process with imperfect maintenance. We define a new class of models, the generalized virtual age (GVA) models, which generalize both the virtual age models for $\mathrm{CM}$ only and the usual competing risks models. A GVA model is defined by a sequence of effective ages which characterizes the effects of both types of maintenance according to a classical virtual age model, and a usual competing risks model which characterizes the dependency between the two types of maintenance.

The paper is organized as follows. The general framework for modelling the PM-CM process is developed in Section 2. In Section 3 we present the usual competing risks model in the PM-CM context. Generalized competing risks models and generalized virtual age models are respectively defined in Sections 4 and 5. In Sections 6 and 7 we present several particular GVA models based on basic assumptions on the virtual ages and the dependency between PM and CM. Finally, in Section 8 we introduce the idea of nonsymmetrical maintenance effects.

\section{General framework for modelling the PM-CM process}

\subsection{Notation and assumptions}

The PM-CM process is the sequence of PM times and CM times. Maintenance durations are assumed to be negligible or not taken into account. The observations are then composed of three point processes, for which we introduce the following notation.

For the global maintenance process (PM and CM), we introduce the processes

- $\left\{C_{i}\right\}_{i \geq 1}$, the maintenance times $\left(C_{0}=0\right)$;

- $\left\{W_{i}\right\}_{i \geq 1}$, the times between maintenances, with $W_{i}=C_{i}-C_{i-1}$;

- $K=\left\{K_{t}\right\}_{t \geq 0}$, the maintenance counting process, with $K_{t}=\sum_{i=1}^{\infty} 1_{\left\{C_{i} \leq t\right\}}$; and

- $\left\{U_{i}\right\}_{i \geq 1}$, the indicators of maintenance types, with

$$
U_{i}= \begin{cases}0 & \text { if the } i \text { th maintenance is a CM } \\ 1 & \text { if the } i \text { th maintenance is a PM. }\end{cases}
$$

For the failure, or CM, process, we introduce the processes

- $\left\{T_{i}\right\}_{i \geq 1}$, the CM times $\left(T_{0}=0\right)$;

- $\left\{X_{i}\right\}_{i \geq 1}$, the times between CMs, with $X_{i}=T_{i}-T_{i-1}$; and

- $N=\left\{N_{t}\right\}_{t \geq 0}$, the CM counting process, with $N_{t}=\sum_{i=1}^{\infty} 1_{\left\{T_{i} \leq t\right\}}$. 


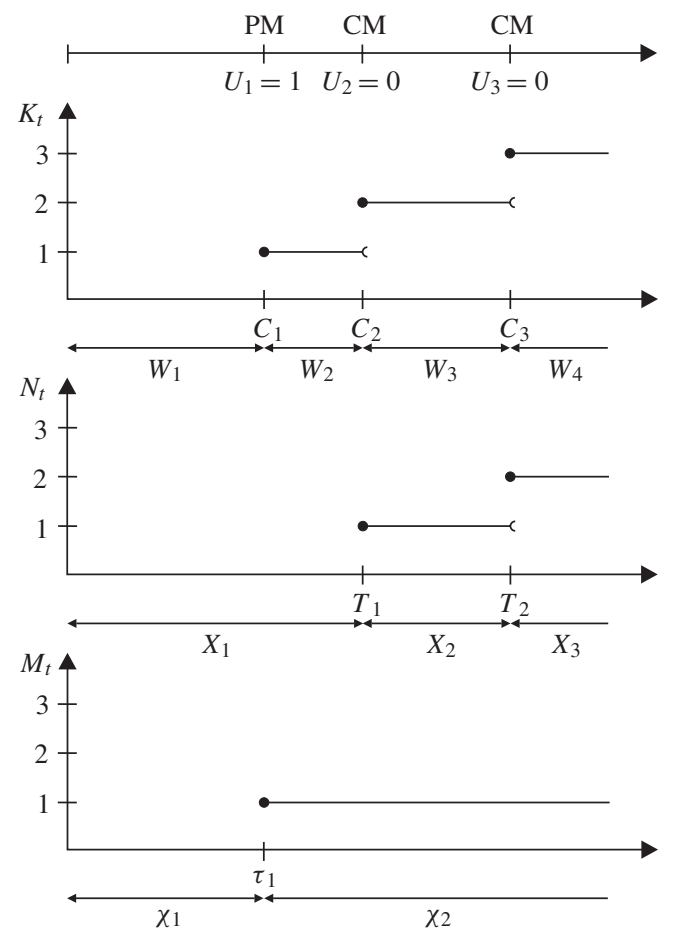

FIGURE 1: A trajectory of the PM-CM process.

Finally, for the PM process, we introduce the processes

- $\left\{\tau_{i}\right\}_{i \geq 1}$, the PM times $\left(\tau_{0}=0\right)$;

- $\left\{\chi_{i}\right\}_{i \geq 1}$, the times between PMs, with $\chi_{i}=\tau_{i}-\tau_{i-1}$; and

- $M=\left\{M_{t}\right\}_{t \geq 0}$, the PM counting process, with $M_{t}=\sum_{i=1}^{\infty} 1_{\left\{\tau_{i} \leq t\right\}}$.

In Figure 1 we present an example of a trajectory of the PM-CM process.

In the following, bold characters denote vectors: for example, $\boldsymbol{T}_{n}=\left(T_{1}, \ldots, T_{n}\right)$.

There are two equivalent representations of the PM-CM process. First, it can be written as a bivariate counting process, $\left\{N_{t}, M_{t}\right\}_{t \geq 0}$. Second, it can be written as a coloured or marked counting process, $\left\{K_{t}, U_{K_{t}}\right\}_{t \geq 0}$. The colour associated with an event of the global maintenance process specifies whether the maintenance is preventive or corrective. The representations are strictly equivalent:

$$
\begin{array}{rlrl}
N_{t}=\sum_{i=1}^{K_{t}}\left(1-U_{i}\right) & \text { and } \quad M_{t} & =\sum_{i=1}^{K_{t}} U_{i}, \quad \text { while } \\
K_{t}=N_{t}+M_{t} & \text { and } \quad U_{k}=M_{C_{k}}-M_{C_{k-1}} .
\end{array}
$$

In order to use the framework of the theory of point processes as in [1], we need to define a filtration. In this paper, $\mathscr{H}_{t}, t \geq 0$, will be the natural filtration generated by the pasts of the processes $N$ and $M$ at time $t$. It can be equivalently written $\mathscr{H}_{t}=\sigma\left(\left\{N_{s}, M_{s}\right\}_{0 \leq s \leq t}\right)=$ $\sigma\left(\left\{K_{s}, U_{K_{s}}\right\}_{0 \leq s \leq t}\right)$. Furthermore, we do not take into account the case where maintenance 
efficiency can depend on external variables, such as in the Brown-Proschan model (this case was studied in [9]).

For a counting process, for example $N, N_{t^{-}}$represents the left-hand limit of $N_{t}$. For the filtration, $\mathscr{H}_{t^{-}}=\bigcup_{s<t} \mathscr{H}_{s}$.

Finally, it is assumed that there cannot be more than one maintenance at the same time:

$$
\mathrm{P}\left(K_{t+\Delta t}-K_{t^{-}}>1 \mid \mathscr{H}_{t^{-}}\right)=o(\Delta t) \text { for all } t \geq 0 \text { and all } \Delta t>0 .
$$

\subsection{Characterization of the PM-CM process}

In order to characterize the $\mathrm{PM}-\mathrm{CM}$ process, let us define three intensities, relative to the whole maintenance history [1, pp. 74-75]. The CM intensity is

$$
\lambda_{t}^{N}=\lim _{\Delta t \rightarrow 0} \frac{1}{\Delta t} \mathrm{P}\left(N_{t+\Delta t}-N_{t^{-}}=1 \mid \mathscr{H}_{t^{-}}\right) .
$$

The PM intensity is

$$
\lambda_{t}^{M}=\lim _{\Delta t \rightarrow 0} \frac{1}{\Delta t} \mathrm{P}\left(M_{t+\Delta t}-M_{t^{-}}=1 \mid \mathscr{H}_{t^{-}}\right) .
$$

The (global) maintenance intensity is

$$
\lambda_{t}^{K}=\lim _{\Delta t \rightarrow 0} \frac{1}{\Delta t} \mathrm{P}\left(K_{t+\Delta t}-K_{t^{-}}=1 \mid \mathscr{H}_{t^{-}}\right) .
$$

Note that the global maintenance intensity is simply the sum of the PM and CM intensities [1, pp. 74-75]:

$$
\lambda_{t}^{K}=\lambda_{t}^{N}+\lambda_{t}^{M}
$$

In the remainder of the paper, we find it more convenient to write $\left(\boldsymbol{W}_{k}, \boldsymbol{U}_{k}\right)$ instead of $\mathscr{H}_{C_{k}}$ in conditional probabilities.

The PM and CM intensities completely characterize the PM-CM process. For instance, the joint and marginal conditional distributions of times between maintenances and maintenance indicators are given as functions of the intensities by Jacod's formulae [1, p. 96]:

$$
\begin{gathered}
\mathrm{P}\left(W_{k+1}>w, U_{k+1}=0 \mid \boldsymbol{W}_{k}, \boldsymbol{U}_{k}\right)=\int_{w}^{\infty} \lambda_{C_{k}+u}^{N} \exp \left(-\int_{0}^{u} \lambda_{C_{k}+s}^{K} \mathrm{~d} s\right) \mathrm{d} u, \\
\mathrm{P}\left(W_{k+1}>w, U_{k+1}=1 \mid \boldsymbol{W}_{k}, \boldsymbol{U}_{k}\right)=\int_{w}^{\infty} \lambda_{C_{k}+u}^{M} \exp \left(-\int_{0}^{u} \lambda_{C_{k}+s}^{K} \mathrm{~d} s\right) \mathrm{d} u, \\
\mathrm{P}\left(W_{k+1}>w \mid \boldsymbol{W}_{k}, \boldsymbol{U}_{k}\right)=\exp \left(-\int_{0}^{w} \lambda_{C_{k}+s}^{K} \mathrm{~d} s\right), \\
\mathrm{P}\left(U_{k+1}=0 \mid \boldsymbol{W}_{k}, \boldsymbol{U}_{k}\right)=\int_{0}^{\infty} \lambda_{C_{k}+u}^{N} \exp \left(-\int_{0}^{u} \lambda_{C_{k}+s}^{K} \mathrm{~d} s\right) \mathrm{d} u .
\end{gathered}
$$

Conversely, the PM and CM intensities are functions of the joint conditional distributions of times between maintenances and maintenance indicators:

$$
\begin{aligned}
& \lambda_{t}^{N}=\frac{-(\mathrm{d} / \mathrm{d} t) \mathrm{P}\left(W_{K_{t^{-}}+1}>t-C_{K_{t^{-}}}, U_{K_{t^{-}}+1}=0 \mid \boldsymbol{W}_{K_{t^{-}}}, \boldsymbol{U}_{K_{t^{-}}}\right)}{\mathrm{P}\left(W_{K_{t^{-}}+1}>t-C_{K_{t^{-}}} \mid \boldsymbol{W}_{K_{t^{-}}}, \boldsymbol{U}_{K_{t^{-}}}\right)}, \\
& \lambda_{t}^{M}=\frac{-(\mathrm{d} / \mathrm{d} t) \mathrm{P}\left(W_{K_{t^{-}}+1}>t-C_{K_{t^{-}}}, U_{K_{t^{-}}+1}=1 \mid \boldsymbol{W}_{K_{t^{-}}}, \boldsymbol{U}_{K_{t^{-}}}\right)}{\mathrm{P}\left(W_{K_{t^{-}}+1}>t-C_{K_{t^{-}}} \mid \boldsymbol{W}_{K_{t^{-}}}, \boldsymbol{U}_{K_{t^{-}}}\right)} .
\end{aligned}
$$


Finally, in a parametric approach, the (vector of) parameters, $\boldsymbol{\theta}$, of the PM and CM intensities can be estimated by the maximum likelihood method. The likelihood function associated with a single observation of the PM-CM process on $[0, t]$ ( $t$ can be a maintenance time but need not be) is $[1$, p. 102]

$$
L_{t}(\boldsymbol{\theta})=\left[\prod_{i=1}^{K_{t}}\left(\lambda_{C_{i}}^{N}\right)^{1-U_{i}}\left(\lambda_{C_{i}}^{M}\right)^{U_{i}}\right] \exp \left(-\sum_{j=1}^{K_{t^{-}+1}} \int_{C_{j-1}}^{C_{j}} \lambda_{s}^{K} \mathrm{~d} s\right) .
$$

Now the problem is to define models which can take into account various kinds of assumption about the efficiency and dependence of each type of maintenance.

\section{Usual competing risks models}

A simple way of modelling the PM-CM process is the competing risks approach, developed in the context of maintenance in [5] and [6].

After the $k$ th maintenance, the latent time to the next failure (or the next $\mathrm{CM}$ ) is a random variable $Z_{k+1}$. However, the failure can be avoided by a potential PM that can take place at a random time, $Y_{k+1}$, after the $k$ th maintenance. Then the real time until the next maintenance is $W_{k+1}=\min \left(Y_{k+1}, Z_{k+1}\right)$ and the maintenance indicator is $U_{k+1}=1_{\left\{Y_{k+1} \leq Z_{k+1}\right\}}$. The random variables $Y_{k+1}$ and $Z_{k+1}$ are called the risk variables.

In this paper, by the usual competing risks approach we mean the classical situation where the couples $\left\{\left(Y_{k}, Z_{k}\right)\right\}_{k \geq 1}$ are independent and identically distributed, so the $\left\{\left(W_{k}, U_{k}\right)\right\}_{k \geq 1}$ are also independent and identically distributed. This means that the effect of every PM and CM is supposed to be perfect. In this situation, the models can be easily studied without using the point process framework developed in the previous section.

The dependency between each type of maintenance is expressed by the joint distribution of $\left(Y_{1}, Z_{1}\right)$, characterized by the joint survival function,

$$
S_{1}(y, z)=\mathrm{P}\left(Y_{1}>y, Z_{1}>z\right) .
$$

The partial derivatives $(\partial / \partial y) S_{1}(y, z),(\partial / \partial z) S_{1}(y, z)$, and $\left(\partial^{2} / \partial y \partial z\right) S_{1}(y, z)$ are assumed to exist.

The main problem of the usual competing risks models is that the joint distribution of $Y_{1}$ and $Z_{1}$ is not identifiable [18]. In fact, the distribution of the observations $\left\{\left(W_{k}, U_{k}\right)\right\}_{k \geq 1}$ depends only on the subsurvival functions [8, p. 4],

$$
\begin{aligned}
& S_{Z_{1}}^{*}(z)=\mathrm{P}\left(Z_{1}>z, Z_{1}<Y_{1}\right)=\mathrm{P}\left(W_{1}>z, U_{1}=0\right), \\
& S_{Y_{1}}^{*}(y)=\mathrm{P}\left(Y_{1}>y, Y_{1} \leq Z_{1}\right)=\mathrm{P}\left(W_{1}>y, U_{1}=1\right),
\end{aligned}
$$

or, equivalently, the subhazard rates [8, p. 10],

$$
\begin{aligned}
\lambda_{\mathrm{c}}(w) & =\lim _{\Delta w \rightarrow 0} \frac{1}{\Delta w} \mathrm{P}\left(w<Z_{1} \leq w+\Delta w, Z_{1}<Y_{1} \mid W_{1}>w\right) \\
& =\lim _{\Delta w \rightarrow 0} \frac{1}{\Delta w} \mathrm{P}\left(w<W_{1} \leq w+\Delta w, U_{1}=0 \mid W_{1}>w\right), \\
\lambda_{\mathrm{p}}(w) & =\lim _{\Delta w \rightarrow 0} \frac{1}{\Delta w} \mathrm{P}\left(w<Y_{1} \leq w+\Delta w, Y_{1} \leq Z_{1} \mid W_{1}>w\right) \\
& =\lim _{\Delta w \rightarrow 0} \frac{1}{\Delta w} \mathrm{P}\left(w<W_{1} \leq w+\Delta w, U_{1}=1 \mid W_{1}>w\right),
\end{aligned}
$$


which can be understood as the hazard rates of the first maintenance time when it is corrective and, respectively, preventive.

Since the marginal survival function of the first time to maintenance satisfies

$$
S_{W_{1}}(w)=\mathrm{P}\left(W_{1}>w\right)=S_{1}(w, w)=S_{Y_{1}}^{*}(w)+S_{Z_{1}}^{*}(w),
$$

the joint survival function and the subhazard rates are linked by the following equations, where a prime denotes (ordinary) differentiation:

$$
\begin{aligned}
& \lambda_{\mathrm{c}}(w)=\frac{-S_{Z_{1}}^{*}{ }^{\prime}(w)}{S_{Y_{1}}^{*}(w)+S_{Z_{1}}^{*}(w)}=\frac{-\left[(\partial / \partial z) S_{1}(y, z)\right]_{(w, w)}}{S_{1}(w, w)}, \\
& \lambda_{\mathrm{p}}(w)=\frac{-S_{Y_{1}}^{*}{ }^{\prime}(w)}{S_{Y_{1}}^{*}(w)+S_{Z_{1}}^{*}(w)}=\frac{-\left[(\partial / \partial y) S_{1}(y, z)\right]_{(w, w)}}{S_{1}(w, w)} .
\end{aligned}
$$

It is obvious that $\lambda_{\mathrm{c}}(t)+\lambda_{\mathrm{p}}(t)=\lambda_{W_{1}}(t)$, the hazard rate of $W_{1}$. Moreover, by Tsiatis's theorem of [18],

$$
\begin{aligned}
& S_{Z_{1}}^{*}(z)=\int_{z}^{\infty}\left[-\frac{\partial}{\partial z} S_{1}(y, z)\right]_{(s, s)} \mathrm{d} s, \\
& S_{Y_{1}}^{*}(y)=\int_{y}^{\infty}\left[-\frac{\partial}{\partial y} S_{1}(y, z)\right]_{(s, s)} \mathrm{d} s .
\end{aligned}
$$

Thus, the distribution of the observations depends on the joint survival function only through its values for $y=z$, which illustrates the identifiability problem mentioned above.

The most usual dependency assumptions for competing risks models related to failure and maintenance are the independent risk assumption and the random sign assumption [6]. The corresponding models will be presented in Section 7.

Regarding our problem, the drawback of competing risks models is that all PMs and CMs are assumed to be AGAN. In the following, we propose to generalize the usual competing risks models, in order to take into account any kind of imperfect maintenance effect.

\section{Generalized competing risks models}

By a generalized competing risks (GCR) model we will mean a competing risks model for which the couples $\left\{\left(Y_{k}, Z_{k}\right)\right\}_{k \geq 1}$ are not assumed to be independent and identically distributed. The couples $\left\{\left(W_{k}, U_{k}\right)\right\}_{k \geq 1}$ are therefore also not independent and identically distributed. Thus, the effect of every PM and CM can be imperfect. The usual competing risks objects are naturally generalized by introducing a conditioning on the past of the PM-CM process.

Definition 1. The (generalized) joint survival functions of the risk variables are

$$
S_{k+1}\left(y, z ; \boldsymbol{W}_{k}, \boldsymbol{U}_{k}\right)=\mathrm{P}\left(Y_{k+1}>y, Z_{k+1}>z \mid \boldsymbol{W}_{k}, \boldsymbol{U}_{k}\right), \quad k \geq 1 .
$$

The (generalized) subsurvival functions are

$$
\begin{aligned}
& S_{Z_{k+1}}^{*}\left(z ; \boldsymbol{W}_{k}, \boldsymbol{U}_{k}\right)=\mathrm{P}\left(Z_{k+1}>z, Z_{k+1}<Y_{k+1} \mid \boldsymbol{W}_{k}, \boldsymbol{U}_{k}\right), \\
& S_{Y_{k+1}}^{*}\left(y ; \boldsymbol{W}_{k}, \boldsymbol{U}_{k}\right)=\mathrm{P}\left(Y_{k+1}>y, Y_{k+1} \leq Z_{k+1} \mid \boldsymbol{W}_{k}, \boldsymbol{U}_{k}\right) .
\end{aligned}
$$


The (generalized) marginal survival functions of the risk variables are

$$
\begin{aligned}
& S_{Z_{k+1}}\left(z ; \boldsymbol{W}_{k}, \boldsymbol{U}_{k}\right)=\mathrm{P}\left(Z_{k+1}>z \mid \boldsymbol{W}_{k}, \boldsymbol{U}_{k}\right), \\
& S_{Y_{k+1}}\left(y ; \boldsymbol{W}_{k}, \boldsymbol{U}_{k}\right)=\mathrm{P}\left(Y_{k+1}>y \mid \boldsymbol{W}_{k}, \boldsymbol{U}_{k}\right) .
\end{aligned}
$$

The partial derivatives of the joint survival functions,

$$
\left(\frac{\partial}{\partial y}\right) S_{k+1}(y, z ; \cdot, \cdot), \quad\left(\frac{\partial}{\partial z}\right) S_{k+1}(y, z ; \cdot, \cdot), \quad \text { and } \quad\left(\frac{\partial^{2}}{\partial y \partial z}\right) S_{k+1}(y, z ; \cdot, \cdot),
$$

are assumed to exist for all $k$. Relations similar to (8) and (9) then hold between the subsurvival functions and joint survival functions:

$$
\begin{aligned}
& S_{Z_{k+1}}^{*}\left(z, \boldsymbol{W}_{k}, \boldsymbol{U}_{k}\right)=\int_{z}^{\infty}\left[-\frac{\partial}{\partial z} S_{k+1}\left(y, z ; \boldsymbol{W}_{k}, \boldsymbol{U}_{k}\right)\right]_{(s, s)} \mathrm{d} s, \\
& S_{Y_{k+1}}^{*}\left(y ; \boldsymbol{W}_{k}, \boldsymbol{U}_{k}\right)=\int_{y}^{\infty}\left[-\frac{\partial}{\partial y} S_{k+1}\left(y, z ; \boldsymbol{W}_{k}, \boldsymbol{U}_{k}\right)\right]_{(s, s)} \mathrm{d} s .
\end{aligned}
$$

This generalized competing risks approach is easily linked with the coloured point process approach, as follows:

$$
\begin{aligned}
S_{k+1}\left(w, w ; \boldsymbol{W}_{k}, \boldsymbol{U}_{k}\right) & =\mathrm{P}\left(W_{k+1}>w \mid \boldsymbol{W}_{k}, \boldsymbol{U}_{k}\right), \\
S_{Z_{k+1}}^{*}\left(w ; \boldsymbol{W}_{k}, \boldsymbol{U}_{k}\right) & =\mathrm{P}\left(W_{k+1}>w, U_{k+1}=0 \mid \boldsymbol{W}_{k}, \boldsymbol{U}_{k}\right), \\
S_{Y_{k+1}}^{*}\left(w ; \boldsymbol{W}_{k}, \boldsymbol{U}_{k}\right) & =\mathrm{P}\left(W_{k+1}>w, U_{k+1}=1 \mid \boldsymbol{W}_{k}, \boldsymbol{U}_{k}\right) .
\end{aligned}
$$

The CM, PM, and global intensities can be written in terms of the PM-CM survival functions:

$$
\begin{aligned}
\lambda_{t}^{N} & =\frac{\left[-(\partial / \partial z) S_{K_{t^{-}}+1}\left(y-C_{K_{t^{-}}}, z-C_{K_{t^{-}}} ; \boldsymbol{W}_{K_{t^{-}}}, \boldsymbol{U}_{K_{t^{-}}}\right)\right]_{(t, t)}}{S_{K_{t^{-}}+1}\left(t-C_{K_{t^{-}}}, t-C_{K_{t^{-}}} ; \boldsymbol{W}_{K_{t^{-}}}, \boldsymbol{U}_{K_{t^{-}}}\right)}, \\
\lambda_{t}^{M} & =\frac{\left[-(\partial / \partial y) S_{K_{t^{-}}+1}\left(y-C_{K_{t^{-}}}, z-C_{K_{t^{-}}} ; \boldsymbol{W}_{K_{t^{-}}}, \boldsymbol{U}_{K_{t^{-}}}\right)\right]_{(t, t)}}{S_{K_{t^{-}}+1}\left(t-C_{K_{t^{-}}}, t-C_{K_{t^{-}}} ; \boldsymbol{W}_{K_{t^{-}}}, \boldsymbol{U}_{K_{t^{-}}}\right)}, \\
\lambda_{t}^{K} & =-\frac{\mathrm{d}}{\mathrm{d} t} \ln S_{K_{t^{-}}+1}\left(t-C_{K_{t^{-}}}, t-C_{K_{t^{-}}} ; \boldsymbol{W}_{K_{t^{-}}}, \boldsymbol{U}_{K_{t^{-}}}\right) .
\end{aligned}
$$

Finally, the likelihood (3) can be rewritten:

$$
\begin{aligned}
L_{t}(\boldsymbol{\theta})= & S_{K_{t^{-}}+1}\left(t-C_{K_{t^{-}}}, t-C_{K_{t^{-}}} ; \boldsymbol{W}_{K_{t^{-}}}, \boldsymbol{U}_{K_{t^{-}}}\right) \\
& \times\left[\prod_{i=1}^{K_{t}}\left[-\frac{\partial}{\partial y} S_{i}\left(y, z ; \boldsymbol{W}_{i-1}, \boldsymbol{U}_{i-1}\right)\right]_{\left(W_{i}, W_{i}\right)}^{U_{i}}\left[-\frac{\partial}{\partial z} S_{i}\left(y, z ; \boldsymbol{W}_{i-1}, \boldsymbol{U}_{i-1}\right)\right]_{\left(W_{i}, W_{i}\right)}^{1-U_{i}}\right] .
\end{aligned}
$$

It can be seen that the PM-CM intensities and the likelihood depend only on the values of the PM-CM survival functions for $y=z$. Therefore, we will here have the same identifiability problem as in the usual competing risks models. To illustrate this problem, let us introduce the notion of conditional independence.

Definition 2. Risk variables $\left\{\left(Y_{k}, Z_{k}\right)\right\}_{k \geq 1}$ are said to be conditionally independent if and only if they are independent conditionally on the past of the PM-CM process, i.e.

$$
S_{k+1}\left(y, z ; \boldsymbol{W}_{k}, \boldsymbol{U}_{k}\right)=S_{Y_{k+1}}\left(y ; \boldsymbol{W}_{k}, \boldsymbol{U}_{k}\right) S_{Z_{k+1}}\left(z ; \boldsymbol{W}_{k}, \boldsymbol{U}_{k}\right)
$$

for all $k \geq 0$, all $y \geq 0$, and all $z \geq 0$. The corresponding GCR model will be called the conditionally independent generalized competing risks (CIGCR) model. 
For GCR models, the following proposition is equivalent to Theorem 1 of [7].

Proposition 1. (i) Two CIGCR models with the same PM and CM intensities have the same generalized joint survival functions.

(ii) For every GCR model, there exists a CIGCR model with the same PM and CM intensities.

Proof. The proof of (i) is obvious. To prove (ii), let $\left\{\left(Y_{k}, Z_{k}\right)\right\}_{k \geq 1}$ be a GCR model and let $\left\{\left(\tilde{Y}_{k}, \tilde{Z}_{k}\right)\right\}_{k \geq 1}$ be a CIGCR model. Since $\tilde{\boldsymbol{Y}}_{k}$ and $\tilde{\boldsymbol{Z}}_{k}$ are conditionally independent, (10) implies that

$$
\lambda_{t}^{\tilde{N}}=\frac{-(\mathrm{d} / \mathrm{d} t) S_{\tilde{Z}_{K_{t^{-}}+1}}\left(t-C_{K_{t^{-}}} ; \boldsymbol{W}_{K_{t^{-}}}, \boldsymbol{U}_{K_{t^{-}}}\right)}{S_{\tilde{Z}_{K_{t^{-}}+1}}\left(t-C_{K_{t^{-}}} ; \boldsymbol{W}_{K_{t^{-}}}, \boldsymbol{U}_{K_{t^{-}}}\right)} .
$$

Therefore,

$$
S_{\tilde{Z}_{k+1}}\left(z ; \boldsymbol{W}_{k}, \boldsymbol{U}_{k}\right)=\exp \left(-\int_{0}^{z} \lambda_{C_{k}+v}^{\tilde{N}} \mathrm{~d} v\right),
$$

so conditionally independent risk variables such that

$$
\begin{aligned}
& S_{\tilde{Z}_{k+1}}\left(z ; \boldsymbol{W}_{k}, \boldsymbol{U}_{k}\right)=\exp \left(-\int_{0}^{z} \lambda_{C_{k}+v}^{N} \mathrm{~d} v\right), \\
& S_{\tilde{Y}_{k+1}}\left(y ; \boldsymbol{W}_{k}, \boldsymbol{U}_{k}\right)=\exp \left(-\int_{0}^{y} \lambda_{C_{k}+v}^{M} \mathrm{~d} v\right),
\end{aligned}
$$

imply that $\lambda_{t}^{\tilde{N}}=\lambda_{t}^{N}$ and $\lambda_{t}^{\tilde{M}}=\lambda_{t}^{M}$. This proves (ii).

Part (i) of the proposition proves that, for a CIGCR model, $S_{k+1}$ is identifiable for all $k$. Part (ii) proves that this is not true for all GCR models.

\section{Generalized virtual age models}

Virtual age models have been introduced by Kijima [12] for systems subject only to CM. The idea of these models is to assume that there exists a sequence of random variables $\left\{A_{k}\right\}_{k \geq 1}$, with $A_{0}=0$, called effective ages, such that after the $k$ th $\mathrm{CM}$ the system behaves like a new one that has survived without failure until $A_{k}$, i.e.

$$
\mathrm{P}\left(X_{k+1}>x \mid A_{k}, X_{k}\right)=\mathrm{P}\left(X>A_{k}+x \mid X>A_{k}, A_{k}\right) \text { for all } x \geq 0,
$$

where $X$ is a random variable independent of $A_{k}$ and with the same distribution as $X_{1}$. In the coloured point process approach, this model can naturally be generalized to describe the $\mathrm{PM}-\mathrm{CM}$ process by assuming that

$$
\begin{aligned}
& \mathrm{P}\left(W_{k+1}>w, U_{k+1}=u \mid \boldsymbol{W}_{k}, \boldsymbol{U}_{k}\right) \\
& \quad=\mathrm{P}\left(W>A_{k}+w, U=u \mid W>A_{k}, A_{k}\right) \quad \text { for all } w \geq 0 \text { and all } u \in\{0,1\}
\end{aligned}
$$

where $(W, U)$ is a random couple with the same distribution as $\left(W_{1}, U_{1}\right)$ and $A_{k}$ is a deterministic function of $\left(\boldsymbol{W}_{k}, \boldsymbol{U}_{k}\right)$ independent of $(W, U)$. Using (1), (2), (4), and (5), the PM and CM intensities can be derived and the model can be defined as follows.

Definition 3. Let $A_{0}=0$ and, for all $k \geq 1$, let $A_{k}$ be a deterministic function of $\left(\boldsymbol{W}_{k}, \boldsymbol{U}_{k}\right)$. A generalized virtual age (GVA) model is a PM-CM process where the PM, CM, and global 
intensities are defined as

$$
\begin{gathered}
\lambda_{t}^{N}=\lambda_{\mathrm{c}}\left(A_{K_{t^{-}}}+t-C_{k_{t^{-}}}\right), \quad \lambda_{t}^{M}=\lambda_{\mathrm{p}}\left(A_{K_{t^{-}}}+t-C_{K_{t^{-}}}\right), \\
\text {and } \lambda_{t}^{K}=\lambda_{W_{1}}\left(A_{K_{t^{-}}}+t-C_{K_{t^{-}}}\right),
\end{gathered}
$$

where $\lambda_{\mathrm{c}}$ and $\lambda_{\mathrm{p}}$ are the subhazard rates of the first latent PM and CM times, defined in (4) and (5). We call $A_{K_{t^{-}}}+t-C_{K_{t^{-}}}$the virtual age of the system at time $t$, while $A_{k}$, the virtual age of the system just after the $k$ th maintenance, is called the effective age at kth maintenance.

The model is then completely characterized by the effective ages and the distribution of $\left(W_{1}, U_{1}\right)$.

It is also possible to build the GVA models using a generalized competing risks approach. With the same sequence $\left\{A_{k}\right\}_{k \geq 0}$, let us assume that after the $k$ th maintenance the risk variables $Y_{k+1}$ and $Z_{k+1}$ behave like the risk variables of a new system, never maintained before $A_{k}$. Then

$$
\mathrm{P}\left(Y_{k+1}>y, Z_{k+1}>z \mid \boldsymbol{W}_{k}, \boldsymbol{U}_{k}\right)=\mathrm{P}\left(Y>A_{k}+y, Z>A_{k}+z \mid Y>A_{k}, Z>A_{k}, A_{k}\right),
$$

where $(Y, Z)$ is a random couple with the same distribution as $\left(Y_{1}, Z_{1}\right)$ and $A_{k}$ is a deterministic function of $\left(\boldsymbol{W}_{k}, \boldsymbol{U}_{k}\right)$ independent of $(Y, Z)$. Note that here the effect of maintenance is symmetrical since the same effective age, $A_{k}$, influences both risk $Y_{k+1}$ and risk $Z_{k+1}$. It is possible to define nonsymmetrical effects, as we will see in Section 8.

The PM-CM survival function is then

$$
S_{k+1}\left(y, z ; \boldsymbol{W}_{k}, \boldsymbol{U}_{k}\right)=\frac{S_{1}\left(A_{k}+y, A_{k}+z\right)}{S_{1}\left(A_{k}, A_{k}\right)}
$$

and, using (10), (11), (12), and (15), we obtain the CM, PM, and global maintenance intensities of the GVA model as in (13) and (14).

The generalized competing risks approach is more complete than the coloured point process approach since it provides an explanation for the distribution of the observations based on modelling considerations on potential times to PM and CM. Unfortunately, the identifiability problem prevents us from validating these additional modelling assumptions using the observations.

The generalized subsurvival functions are

$$
\begin{aligned}
& S_{Z_{k+1}}^{*}\left(z ; \boldsymbol{W}_{k}, \boldsymbol{U}_{k}\right)=\int_{z}^{\infty} \lambda_{\mathrm{c}}\left(A_{k}+s\right) \exp \left(-\int_{0}^{s} \lambda_{W_{1}}\left(A_{k}+u\right) \mathrm{d} u\right) \mathrm{d} s, \\
& S_{Y_{k+1}}^{*}\left(y ; \boldsymbol{W}_{k}, \boldsymbol{U}_{k}\right)=\int_{y}^{\infty} \lambda_{\mathrm{p}}\left(A_{k}+s\right) \exp \left(-\int_{0}^{s} \lambda_{W_{1}}\left(A_{k}+u\right) \mathrm{d} u\right) \mathrm{d} s .
\end{aligned}
$$

Finally, to build a GVA model we must make two more steps.

1. Characterize both PM and CM effects by defining the effective ages $\left\{A_{k}\right\}_{k \geq 1}$ according to a classical virtual age model.

2. Characterize the dependency between PM and CM by defining the joint survival function $S_{1}$ according to a usual competing risks model. Then derive the subhazard rates $\lambda_{\mathrm{c}}$ and $\lambda_{\mathrm{p}}$ from $S_{1}$ using (6) and (7).

In Sections 6 and 7 we present several particular cases of the general model based on basic assumptions on the effects of, and the dependency between, PM and CM. 


\section{Assumptions on the PM and CM effects}

\subsection{Perfect PM and $\mathrm{CM}$}

Here each maintenance is assumed to be AGAN, i.e. it perfectly repairs the system and leaves it as if it were brand new. The effective ages are then equal to 0 . The maintenance process $K$ is a renewal process but the failure process is, in general, not:

$$
\lambda_{t}^{N}=\lambda_{\mathrm{c}}\left(t-C_{K_{t^{-}}}\right) \quad \text { and } \quad \lambda_{t}^{K}=\lambda_{W_{1}}\left(t-C_{K_{t^{-}}}\right) .
$$

This model corresponds to the usual competing risks model.

\subsection{Minimal PM and $\mathrm{CM}$}

Here each maintenance is assumed to be ABAO, i.e. it restores the system to the state it was in just before the maintenance action. The effective ages are thus equal to the last maintenance times, i.e. $A_{k}=C_{k}$, and, so, the CM, PM, and global maintenance intensities are simply functions of time:

$$
\lambda_{t}^{N}=\lambda_{\mathrm{c}}(t)
$$

The CM, PM, and global maintenance processes are nonhomogeneous Poisson processes.

\section{3. $\mathrm{ARA}_{\infty}$ model for $\mathrm{PM}$ and $\mathrm{CM}$}

The idea of the model proposed for CM only by Brown et al. [3], also known as the ARA ${ }_{\infty}$ model [10], is to assume that the effect of maintenance is to reduce the virtual age by an amount proportional to its value just before the maintenance. When both PM and CM are present and when their effects are assumed to be similar, this assumption leads to the expression

$$
A_{k}=(1-\rho)\left(A_{k-1}+W_{k}\right)
$$

The model can be generalized to different PM and CM effects by assuming that there are two different reduction factors: one, $\rho_{\mathrm{p}}$, corresponding to PM and one, $\rho_{\mathrm{c}}$, corresponding to $\mathrm{CM}$. Then

$$
A_{k}= \begin{cases}\left(1-\rho_{\mathrm{p}}\right)\left[A_{k-1}+W_{k}\right] & \text { if } U_{k}=1 \\ \left(1-\rho_{\mathrm{c}}\right)\left[A_{k-1}+W_{k}\right] & \text { if } U_{k}=0 .\end{cases}
$$

For $1 \leq j \leq K_{t}$, between $C_{j-1}$ and $t$ there are $M_{t}-M_{C_{j-1}}$ PMs and $N_{t}-N_{C_{j-1}}$ CMs. The failure intensity is thus

$$
\lambda_{t}^{N}=\lambda_{\mathrm{c}}\left(t-C_{K_{t^{-}}}+\sum_{j=1}^{K_{t^{-}}}\left(1-\rho_{\mathrm{p}}\right)^{M_{t^{-}}-M_{C_{j-1}}}\left(1-\rho_{\mathrm{c}}\right)^{N_{t^{-}}-N_{C_{j-1}}} W_{j}\right)
$$

For $\rho_{\mathrm{p}}=\rho_{\mathrm{c}}=\rho$, this reads

$$
\lambda_{t}^{N}=\lambda_{\mathrm{c}}\left(t-\rho \sum_{j=0}^{K_{t^{-}-1}}(1-\rho)^{j} C_{K_{t^{-}}-j}\right)
$$

which is similar to the intensity of the basic ARA $\infty$ model [10]. The cases $\rho_{\mathrm{p}}=1, \rho_{\mathrm{c}}=1$, $\rho_{\mathrm{p}}=0$, and $\rho_{\mathrm{c}}=0$ correspond respectively to AGAN PM, AGAN CM, ABAO PM, and ABAO $\mathrm{CM}$. 


\section{4. $\mathrm{ARA}_{1}$ model for $P M$ and $C M$}

The idea of the proportional age reduction model [13], also known as the $\mathrm{ARA}_{1}$ model [10] for CM only, is to assume that maintenance does not reduce the global virtual age but only the supplement of age gained since the last maintenance. When both PM and CM are present and when their effects are assumed to be similar, this assumption leads to the expression

$$
A_{k}=A_{k-1}+(1-\rho) W_{k}=(1-\rho) C_{k} .
$$

We then have

$$
\lambda_{t}^{N}=\lambda_{\mathrm{c}}\left(t-\rho C_{K_{t^{-}}}\right) .
$$

6.4.1. First model. A first way to generalize the $\mathrm{ARA}_{1}$ model to different $\mathrm{PM}$ and $\mathrm{CM}$ effects is to make an assumption similar to (16) with different maintenance efficiency parameters $\rho_{\mathrm{p}}$ and $\rho_{\mathrm{c}}$, namely

$$
A_{k}= \begin{cases}A_{k-1}+\left(1-\rho_{\mathrm{p}}\right) W_{k} & \text { if } U_{k}=1 \\ A_{k-1}+\left(1-\rho_{\mathrm{c}}\right) W_{k} & \text { if } U_{k}=0\end{cases}
$$

Then the effective age is

$$
A_{k}=\sum_{i=1}^{k}\left(1-\rho_{\mathrm{p}}\right)^{U_{i}}\left(1-\rho_{\mathrm{c}}\right)^{1-U_{i}} W_{i}=C_{k}-\sum_{i=1}^{k} \rho_{\mathrm{p}}^{U_{i}} \rho_{\mathrm{c}}^{1-U_{i}} W_{i}
$$

and the $\mathrm{CM}$ intensity is

$$
\lambda_{t}^{N}=\lambda_{\mathrm{c}}\left(t-\sum_{i=0}^{K_{t^{-}}} \rho_{\mathrm{p}}^{U_{i+1}} \rho_{\mathrm{c}}^{1-U_{i+1}} W_{i+1}\right) .
$$

For $\rho_{\mathrm{p}}=\rho_{\mathrm{c}}=\rho$, the CM intensity is (17). Having $\rho_{\mathrm{c}}=0$ and $\rho_{\mathrm{p}}=0$ respectively imply having $\mathrm{ABAO} \mathrm{CM}$ and $\mathrm{ABAO} \mathrm{PM}$. However, having $\rho_{\mathrm{p}}=1$ corresponds to having PM that is not AGAN but, rather, as good as previous: PM restores the system to the state it was in just after the previous CM ( $A_{k}$ equals $A_{K_{T_{N}} C_{k}}$ rather than 0 ). Thus, PM cannot prevent the ageing due to CM. Similarly, having $\rho_{\mathrm{c}}=1$ does not correspond to having AGAN CM.

6.4.2. Second model. Another generalization of the $\mathrm{ARA}_{1}$ model is to assume that the virtual age after a PM is equal to the virtual age just after the previous PM plus $\left(1-\rho_{\mathrm{p}}\right)$ times the time elapsed between these two PMs. The effect of CM is the same as in the previous model. Here the effective ages satisfy

$$
A_{k}= \begin{cases}A_{K_{\tau_{M_{C}}-1}}+\left(1-\rho_{\mathrm{p}}\right) \chi_{M_{C_{k}}} & \text { if } U_{k}=1, \\ A_{k-1}+\left(1-\rho_{\mathrm{c}}\right) W_{k} & \text { if } U_{k}=0 .\end{cases}
$$

It can be proved recursively that if $U_{k}=1$ then

$$
A_{k}=\left(1-\rho_{\mathrm{p}}\right) C_{k},
$$

and that if $U_{k}=0$ then

$$
A_{k}=A_{K_{\tau_{M_{k}}}}+\left(1-\rho_{\mathrm{c}}\right)\left[C_{k}-\tau_{M_{C_{k}}}\right]
$$


Both of these expressions imply, for all values of $U_{k}$, that

$$
A_{k}=\left(1-\rho_{\mathrm{c}}\right)\left[C_{k}-\tau_{M_{C_{k}}}\right]+\left(1-\rho_{\mathrm{p}}\right) \tau_{M_{C_{k}}} .
$$

The CM intensity is simply

$$
\lambda_{t}^{N}=\lambda_{\mathrm{c}}\left(t-\rho_{\mathrm{c}}\left(C_{K_{t^{-}}}-\tau_{M_{t^{-}}}\right)-\rho_{\mathrm{p}} \tau_{M_{t^{-}}}\right) .
$$

In this model, CM is less efficient than PM, since CM influences failure intensity (and, thus, failure probability) only up to the next PM. This is rather realistic.

Having $\rho_{\mathrm{c}}=1$ still does not correspond to having AGAN CM, but here having $\rho_{\mathrm{p}}=1$ does correspond to having AGAN PM. Having $\rho_{\mathrm{c}}=0$ and $\rho_{\mathrm{p}}=0$ respectively imply having ABAO $\mathrm{CM}$ and ABAO PM.

\subsection{Jack model for PM and CM}

The maintenance efficiency assumptions proposed by Jack [11] in the case of PM planned at deterministic times can also be used in the case of condition-based PM. These assumptions are very similar to that of the previous model. The virtual age after a PM is assumed to be equal to the virtual age just after the previous PM plus $\left(1-\rho_{\mathrm{p}}\right)$ times the virtual age gained (instead of the time elapsed, as in the previous model) between these two PMs. The effect of CM is the same as in the previous model. If $C_{k}$ is a PM time, the virtual age gained since the last PM is

$$
W_{k}+A_{k-1}-A_{K_{\tau_{M_{k}}-1}}
$$

The effective ages thus satisfy

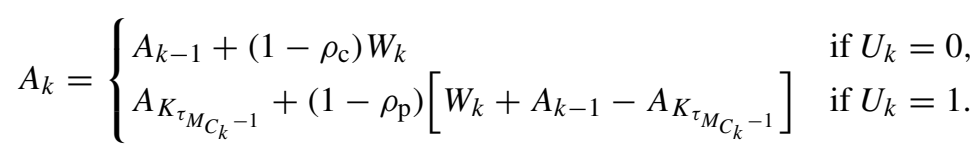

It can be proved [9] that the CM intensity of this model is

$$
\lambda_{t}^{N}=\lambda_{\mathrm{c}}\left(t-\rho_{\mathrm{c}} C_{K_{t^{-}}}+\left(\rho_{\mathrm{c}}-\rho_{\mathrm{p}}\right) \tau_{M_{t^{-}}}-\left(1-\rho_{\mathrm{p}}\right) \rho_{\mathrm{c}} \sum_{j=0}^{M_{C_{K_{t^{-}}}}{ }^{-1}}\left[C_{K_{\tau_{j+1}}-1}-\tau_{j}\right]\right) .
$$

By taking $\rho_{\mathrm{p}}=\rho_{\mathrm{c}}=\rho$ we do not obtain (16). Since the effect of CM is the same as that in the previous model, having $\rho_{\mathrm{c}}=1$ still does not correspond to having AGAN CM. However, having $\rho_{\mathrm{p}}=1$ corresponds to having AGAN PM, and having $\rho_{\mathrm{c}}=0$ and $\rho_{\mathrm{p}}=0$ respectively imply having ABAO CM and ABAO PM.

\section{Assumptions on the dependency between PM and CM}

\subsection{Independent risks model}

The most basic assumption in the usual competing risks models is to consider $Z_{1}$ and $Y_{1}$ to be independent. Such a model is completely defined by the marginal distributions of $Y_{1}$ and $Z_{1}$, and $\lambda_{\mathrm{c}}$ and $\lambda_{\mathrm{p}}$ are then respectively equal to the hazard rates of $Z_{1}$ and $Y_{1}$ :

$$
\lambda_{\mathrm{c}}(t)=\frac{-S_{Z_{1}}^{\prime}(t)}{S_{Z_{1}}(t)} \quad \text { and } \quad \lambda_{\mathrm{p}}(t)=\frac{-S_{Y_{1}}^{\prime}(t)}{S_{Y_{1}}(t)} .
$$


In a parametric approach, it is possible to choose, for example, a Weibull distribution for $Y_{1}$ and $Z_{1}$, in which case $\lambda_{\mathrm{c}}(t)=\alpha_{\mathrm{c}} \beta_{\mathrm{c}} t^{\beta_{\mathrm{c}}-1}$ and $\lambda_{\mathrm{p}}(t)=\alpha_{\mathrm{p}} \beta_{\mathrm{p}} t^{\beta_{\mathrm{p}}-1}$ with $\alpha_{\mathrm{c}}>0, \alpha_{\mathrm{p}}>0, \beta_{\mathrm{c}} \geq 1$, and $\beta_{\mathrm{p}} \geq 1$.

The assumption of independence between the risk variables is not realistic for conditionbased PM, because potential PM times and latent CM times both depend on the degradation of the system. However, using (15), it is clear that the risk variables of a GVA model are conditionally independent if and only if $Z_{1}$ and $Y_{1}$ are independent. Thus, for any GVA model, there exists an equivalent GVA model with independent risks. Even if the assumption of independence is not realistic, the corresponding GVA model can be useful.

\subsection{The repair alert model}

For dependent competing risks, the most usual assumption is the random sign assumption [7], namely that the sign of $Y_{1}-Z_{1}$ is independent of $Z_{1}$. This is equivalent to saying that $U_{1}$ and $Z_{1}$ are independent. Hence, the type of the next maintenance does not depend on the next latent failure time. Another assumption on $Y_{1}$ is needed to completely define the model. For instance, in the repair alert model [16] it is assumed that there exists a nondecreasing function $G(t)$, with $G(0)=0$, such that

$$
\mathrm{P}\left(Y_{1} \leq y \mid Z_{1}=z, Y_{1} \leq Z_{1}\right)=\frac{G(y)}{G(z)} .
$$

The function $G$ characterizes how the maintenance team manages to optimize PM, i.e. it measures the nearness between PM and latent failure times.

Lindqvist et al. [16] proved that

$$
S_{Z_{1}}^{*}(t)=(1-q) S_{Z_{1}}(t) \quad \text { and } \quad S_{Y_{1}}^{*}(t)=q\left[S_{Z_{1}}(t)-G(t) K(t)\right],
$$

where $q=\mathrm{P}\left(U_{1}=1\right)$,

$$
K(t)=\int_{t}^{\infty} \frac{f_{Z_{1}}(x)}{G(x)} \mathrm{d} x,
$$

and $f_{Z_{1}}$ is the density of $Z_{1}$. Let $g$ denote the derivative of $G$. Then

$$
\begin{gathered}
S_{Z_{1}}^{*}{ }^{\prime}(t)=-(1-q) f_{Z_{1}}(t), \quad S_{Y_{1}}^{*}(t)=-q g(t) K(t), \\
S_{1}(t, t)=S_{Z_{1}}(t)-q G(t) K(t),
\end{gathered}
$$

and, finally,

$$
\begin{aligned}
& \lambda_{\mathrm{c}}(t)=\frac{(1-q) f_{Z_{1}}(t)}{S_{Z_{1}}(t)-q G(t) K(t)}, \\
& \lambda_{\mathrm{p}}(t)=\frac{q g(t) K(t)}{S_{Z_{1}}(t)-q G(t) K(t)} .
\end{aligned}
$$

In a parametric approach, the first time to latent failure can have a Weibull distribution $\left(\lambda_{Z_{1}}(t)=\alpha \beta t^{\beta-1}\right.$ with $\alpha>0$ and $\left.\beta \geq 1\right)$ and $G(t)=t^{b}$ with $b>0$. If $b$ is small, PM is likely to be done very early relative to the latent failure time, which is not desirable. If $b$ is large enough, PM is likely to be done just before the latent failure time, so PM is ideal with regards to maintenance costs. As in the independent risks model, four parameters have to be estimated. 
In the intensity proportional repair alert model [14], [15], $G(t)=\Lambda_{Z_{1}}(t)$, the cumulative hazard rate of $Z_{1}$. In this case, there is one fewer parameter to estimate and $K(t)=\operatorname{Ie}\left(\Lambda_{Z_{1}}(t)\right)$, where $\operatorname{Ie}(t)=\int_{t}^{\infty}[\exp (-s) / s] \mathrm{d} s$. Langseth and Lindqvist [14] have applied this model with virtual ages of the Brown-Proschan type. This model involves external variables, so the likelihood (3) cannot be used.

\subsection{The proportional hazards model}

Let us assume that maintenance type $U_{1}$ is independent of maintenance time $W_{1}$. This assumption is quite similar to the random sign assumption. Let $q=\mathrm{P}\left(U_{1}=1\right)$ denote the probability that the first maintenance is preventive. The subsurvival functions are then

$$
S_{Y_{1}}^{*}(y)=q S_{1}(y, y) \quad \text { and } \quad S_{Z_{1}}^{*}(z)=(1-q) S_{1}(y, y),
$$

and the subhazard rates are

$$
\lambda_{\mathrm{c}}(t)=(1-q) \lambda_{W_{1}}(t) \quad \text { and } \quad \lambda_{\mathrm{p}}(t)=q \lambda_{W_{1}}(t) .
$$

This is known as the proportional hazards model [8, p. 12]. With a Weibull model for the first maintenance time $\left(\lambda_{W_{1}}(t)=\alpha \beta t^{\beta-1}\right.$ with $\alpha>0$ and $\left.\beta \geq 1\right)$, there is one fewer parameter to estimate than in the independent risks model.

\section{Nonsymmetrical maintenance effect on the risk variables}

In the GVA model described above, we have assumed that the effect on the risk variables is symmetrical. However, it is possible to define more general models by assuming that there exist two sequences, $\left\{A_{k}^{\mathrm{p}}\right\}_{k \geq 0}$ and $\left\{A_{k}^{\mathrm{c}}\right\}_{k \geq 0}$ with $A_{0}^{\mathrm{p}}=A_{0}^{\mathrm{c}}=0$, such that

$$
\begin{aligned}
& \mathrm{P}\left(Y_{k+1}>y, Z_{k+1}>z \mid \boldsymbol{W}_{k}, \boldsymbol{U}_{k}\right) \\
& \quad=\mathrm{P}\left(Y>A_{k}^{\mathrm{p}}+y, Z>A_{k}^{\mathrm{c}}+z \mid Y>A_{k}^{\mathrm{p}}, Z>A_{k}^{\mathrm{c}}, A_{k}^{\mathrm{p}}, A_{k}^{\mathrm{c}}\right),
\end{aligned}
$$

where $(Y, Z)$ is a random couple with the same distribution as $\left(Y_{1}, Z_{1}\right)$ and $A_{k}^{\mathrm{p}}$ and $A_{k}^{\mathrm{c}}$ are deterministic functions of $\left(\boldsymbol{W}_{k}, \boldsymbol{U}_{k}\right)$ independent of $(Y, Z)$. The idea is to consider maintenances that have different effects on the potential times to the next PM and CM.

Similarly to in Section 5, the PM, CM, and global intensities can be derived as

$$
\begin{aligned}
\lambda_{t}^{N} & =\frac{-\left[(\partial / \partial z) S_{1}\left(A_{K_{t^{-}}}^{\mathrm{p}}+y-C_{K_{t^{-}}}, A_{K_{t^{-}}}^{\mathrm{c}}+z-C_{K_{t^{-}}}\right)\right]_{(t, t)}}{S_{1}\left(A_{K_{t^{-}}}^{\mathrm{p}}+t-C_{K_{t^{-}}}, A_{K_{t^{-}}}^{\mathrm{c}}+t-C_{K_{t^{-}}}\right)}, \\
\lambda_{t}^{M} & =\frac{-\left[(\partial / \partial y) S_{1}\left(A_{K_{t^{-}}}^{\mathrm{p}}+y-C_{K_{t^{-}}}, A_{K_{t^{-}}}^{\mathrm{c}}+z-C_{K_{t^{-}}}\right)\right]_{(t, t)}}{S_{1}\left(A_{K_{t^{-}}}^{\mathrm{p}}+t-C_{K_{t^{-}}}, A_{K_{t^{-}}}^{\mathrm{c}}+t-C_{K_{t^{-}}}\right)}, \\
\lambda_{t}^{K} & =-\frac{\mathrm{d}}{\mathrm{d} t} \ln S_{1}\left(A_{K_{t^{-}}}^{\mathrm{p}}+t-C_{K_{t^{-}}}, A_{K_{t^{-}}}^{\mathrm{c}}+t-C_{K_{t^{-}}}\right) .
\end{aligned}
$$

Note that $A_{K_{t^{-}}}^{\mathrm{p}}+t-C_{K_{t^{-}}}$is not necessarily equal to $A_{K_{t^{-}}}^{\mathrm{c}}+t-C_{K_{t^{-}}}$. Thus, in this general virtual age model, it is probable that $S_{1}$ will be identifiable.

In a different context, Bedford and Lindqvist [2] have proposed a model that can be considered similar to a particular case of this general model. Their idea is equivalent to assuming that each type of maintenance renews (AGAN) its own risk process and leaves the other one in the same state (ABAO), whence

$$
A_{k}^{\mathrm{p}}=C_{k}-\tau_{M_{C_{k}}} \quad \text { and } \quad A_{k}^{\mathrm{c}}=C_{k}-T_{N_{C_{k}}} .
$$


Here $A_{k}^{\mathrm{p}}$ represents the time elapsed since the last PM and $A_{k}^{\mathrm{c}}$ the time elapsed since the last CM. The CM and PM intensities are

$$
\begin{aligned}
\lambda_{t}^{N} & =\frac{-\left[(\partial / \partial z) S_{1}\left(y-\tau_{M_{t^{-}}}, z-T_{N_{t^{-}}}\right)\right]_{(t, t)}}{S_{1}\left(t-\tau_{M_{t^{-}}}, t-T_{N_{t^{-}}}\right)}, \\
\lambda_{t}^{M} & =\frac{-\left[(\partial / \partial y) S_{1}\left(y-\tau_{M_{t^{-}}}, z-T_{N_{t^{-}}}\right)\right]_{(t, t)}}{S_{1}\left(t-\tau_{M_{t^{-}}}, t-T_{N_{t^{-}}}\right)} .
\end{aligned}
$$

In [2] Bedford and Lindqvist proved that, under sensible assumptions, $S_{1}$ is completely identifiable in this model. Their proof is equivalent to proving that $\left(t-\tau_{M_{t^{-}}}, t-T_{N_{t^{-}}}\right)$has some chance of exploring the whole $\mathbb{R}_{+}^{2}$ space for all $t \geq 0$. The distribution of the PM-CM process will thus depend on the values of $S_{1}(y, z)$ for all $(y, z) \in \mathbb{R}^{2}$. A similar method can probably be used to prove the identifiability of the general model defined in (18) and (19).

\section{References}

[1] Andersen, P. K., Borgan, Ø., Gill, R. D. And Keiding, N. (1993). Statistical Models Based on Counting Processes. Springer, New York.

[2] BedFord, T. AND LindQvist, B. H. (2004). The identifiable problem for repairable systems subject to competing risks. Adv. Appl. Prob. 36, 774-790.

[3] Brown, J. F., Mahoney, J. F. and Sivalzian, B. D. (1998). Hysteresis repair in discounted replacement problems. IIE Trans. 15, 156-165.

[4] Brown, M. and Proschan, F. (1983). Imperfect repair. J. Appl. Prob. 20, 851-859.

[5] Bunea, C. AND BEDFord, T. (2002). The effect of model uncertainty on maintenance optimization. IEEE Trans. Reliab. 51, 486-493.

[6] Cooke, R. And Bedford, T. (2002). Reliability databases in perspective. IEEE Trans. Reliab. 51, 294-310.

[7] Cooke, R. M. (1993). The total time test statistic and age-dependent censoring. Statist. Prob. Lett. 18, $307-312$.

[8] Crowder, M. J. (2001). Classical Competing Risks. CRC, Boca Raton, FL.

[9] Doyen, L. (2004). Modélisation et évaluation de l'efficacité de la maintenance des systèmes réparables. Doctoral Thesis, Institut National Polytechnique de Grenoble. Available at http://tel.ccsd.cnrs.fr/documents/ archives0/00/00/77/22/index.html.

[10] Doyen, L. And Gaudoin, G. (2004). Classes of imperfect repair models based on reduction of failure intensity or virtual age. Reliab. Eng. System Safety 84, 45-56.

[11] JACK, N. (1998). Age-reduction models for imperfect maintenance. IMA J. Math. Appl. Business Industry 9 , 347-354.

[12] KiJima, M. (1989). Some results for repairable systems with general repair. J. Appl. Prob. 26, 89-102.

[13] KiJima, M., Morimura, H. AND SuzuKI, Y. (1988). Periodical replacement problem without assuming minimal repair. Operat. Res. 37, 194-203.

[14] Langseth, H. ANd Lindqvist, B. H. (2003). A maintenance model for components exposed to several failure mechanisms and imperfect repair. In Mathematical and Statistical Methods in Reliability, eds K. Doksum and B. H. Lindqvist, World Scientific, River Edge, NJ, pp. 415-430.

[15] Langseth, H. And Lindevist, B. H. (2006). Competing risks for repairable systems: a data study. J. Statist. Planning Infer. 136, 1687-1700.

[16] Lindevist, B. H., Støve, B. And Langseth, H. (2006). Modelling of dependence between critical failure and preventive maintenance: the repair alert model. J. Statist. Planning Infer. 136, 1701-1717.

[17] Pham, H. and Wang, H. (1996). Imperfect maintenance. Europ. J. Operat. Res. 94, 425-438.

[18] Tsiatis, A. (1975). A nonidentifiability aspect of the problem of competing risks. Proc. Nat. Acad. Sci. USA 72, 20-22. 